\title{
Antibacterial and antibiotic-potentiation activities of the methanol extract of some cameroonian spices against Gram-negative multi-drug resistant phenotypes
}

\author{
Igor K Voukeng ${ }^{1}$, Victor Kuete ${ }^{1 *}$, Jean P Dzoyem¹, Aimé G Fankam', Jaures A K Noumedem', Jules R Kuiate ${ }^{1}$ \\ and Jean-Marie Pages ${ }^{2}$
}

\begin{abstract}
Background: The present work was designed to evaluate the antibacterial properties of the methanol extracts of eleven selected Cameroonian spices on multi-drug resistant bacteria (MDR), and their ability to potentiate the effect of some common antibiotics used in therapy.

Results: The extract of Cinnamomum zeylanicum against Escherichia coli ATCC 8739 and AG100 strains showed the best activities, with the lowest minimal inhibitory concentration (MIC) of $64 \mu \mathrm{g} / \mathrm{ml}$. The extract of Dorstenia psilurus was the most active when tested in the presence of an efflux pump inhibitor, phenylalanine Arginine- $\beta$ -

Naphtylamide (PABN), a synergistic effect being observed in $56.25 \%$ of the tested bacteria when it was combined with Erythromycin (ERY).

Conclusion: The present work evidently provides information on the role of some Cameroonian spices in the fight against multi-resistant bacteria.
\end{abstract}

Keywords: Multi-Drug Resistant bacteria, Spices, Methanol extract, Cameroon

\section{Background}

Infectious diseases are one of the leading causes of morbidity and mortality worldwide, especially in developing countries $[1,3]$. Following the massive use of antibiotics in human therapy, bacteria have developed several resistance mechanisms including the efflux of antibiotics [3]. Several Cameroonian spices are known to possess medicinal values [4]. In our previous report, we demonstared that several medicinal spices inhibited the growth of MDR bacteria and were also able to improve the activity of commonly used antibiotics [5]. In our continuous search of antimicrobial drugs from medicinal plant, we designed the present work to investigate the antibacterial potential against Gram-negative MDR bacteria of some of the commonly used medicinal spices in Cameroon

\footnotetext{
* Correspondence: kuetevictor@yahoo.fr

'Department of Biochemistry, Faculty of science, University of Dschang, P.O. Box 67, Dschang, Cameroon, Africa

Full list of author information is available at the end of the article
}

such as Aframomum citratum (Pereira) K. Schum. (Zingiberaceae), Aframomum melegueta (Roscoe) K. Schum. (Zingiberaceae), Scorodophloeus zenkeri Harms (Caesalpiniaceae), Tetrapleura tetraptera (Schum. \& Thonn) Taub. (Mimosaceae), Fagara leprieurii (Guill and Perr) Engl. (Rutaceae), Monodora myristica Dunal (Annonaceae), Piper guineense (Schum and Thonn) (Piperaceae), Dorstenia psilurus Welwitch (Moraceae), Imperata cylindricum Beauv. var. koenigii Durand and Schinz (Gramineae), Pentadiplandra brazzeana Baill. (Capparaceae) and Cinnamomum zeylanicum (Linn) Cor. (Lauraceae).

\section{Material and methods}

Plant materials and extraction

The eleven edible spices used in this work were purchased from Dschang local market, West Region of Cameroon in January 2010. The collected spices material were the fruits of Aframomum citratum, Aframomum melegueta, Scorodophloeus zenkeri, Tetrapleura tetraptera, 
Table 1 Spices used in the present study and evidence of their activities

\begin{tabular}{|c|c|c|c|c|}
\hline Spice samples (Family) & $\begin{array}{l}\text { Herbarium Voucher } \\
\text { number }^{\mathrm{a}}\end{array}$ & Traditional Treatment & Part used & $\begin{array}{l}\text { Bioactive (or potentially active) compounds }{ }^{b} \\
\text { and screened activity }{ }^{c} \text { for crude plant extract }\end{array}$ \\
\hline $\begin{array}{l}\text { Aframomum citratum } \\
\text { (Pereira) K. Schum. } \\
\text { (Zingiberaceae) }\end{array}$ & 37 736/HNC & $\begin{array}{l}\text { Malaria, aphrodisiac, cancer } \\
{[4,6]}\end{array}$ & $\begin{array}{l}\text { Fruits. leaves. } \\
\text { seeds }\end{array}$ & $\frac{\text { Antimicrobial: Ethylacetate extract of fruits on Ec. }}{P a . S a[7]}$ \\
\hline
\end{tabular}

Aframomum melegueta 39 065/HNC (Roscoe) K. Schum.

(Zingiberaceae)

Cinnamomum zeylanicum 22 309/SRFC (Linn) Cor. (Lauraceae)

Dorstenia psilurus

44 839/HNC

Welwitch (Moraceae)

Fagara leprieurii (Guill

37 632/HNC

(Rutaceae) and Perr) Engl.

Malaria, dysentery, carminative, dysmenorrheal, fertility, rubella, leprosy, cancer $[6,8]$

Cancer [4]

Snake bite, rheumatism, head and stomach ache, hypertension, cancer $[4,12,13]$.

Gastritis, gingivitis. bilharzias, Bark, leaves. roots antidiarrhoeal, cancer, laxative, antimicrobial, ulcer gonorrhea, kidney ache. sterility $[4,14,15]$

30 139/SRFC

Imperata cylindricum

Beauv. var. koenigii

Durand et Schinz

(ramineae)

Monodora myristica Dunal (Annonaceae)

2 949/SRFC

Pentadiplandra brazzeana 42 918/HNC Baill. (Capparaceae)

Piper quineense (Schum 6 018/SRFC and Thonn) (Piperaceae)

Scorodophloeus zenkerii 44 803/HNC Harms (Caesalpiniaceae)
Diuretic, anti-inflammatory, Leaves, roots dysentery, urinary tract infections, cancer $[4,16,17]$

Insecticidal, diuretic, hemorrhage, headache, wounds, worm infections, cancer $[4,15,18,19]$

Gastric ulcer, cancer $[4,20] \quad$ Fruits, leaves

Cough, bronchitis, rheumatism, insecticidal, anemia, carminative, stomach ache, cancer $[4,8,21]$

Cancer [4] Leaves, roots constipation, anti-
Cytotoxicity of fruits crude methanol extract [weak activity on leukemia CCRF-CEM and CEM/ADR5000 cells, and pancreatic MiaPaCa-2 cell lines] [4]

Fruits, leaves

Antimicrobial : Aqueous and ethanol extract of leaves on Fo. An [9] Methanol extract of fruits (Q) on Sa. Bs. Ec. Pa. Ca [8]

Cytotoxicity of fruits crude methanol extract [weak activity on leukemia CCRF-CEM and pancreatic MiaPaCa-2 cell lines and significant activity on CEM/ADR5000 cells with $\mid C_{50}$ value of $\left.7.08 \mathrm{\mu g} / \mathrm{ml}\right]$

Fruits, leaves. bark Antimicrobial : Cd, Cm, Lt, Fp [10,11]

Cytotoxicity of leaves crude methanol extract [weak activity on leukemia CCRF-CEM and CEM/ ADR5000 cells, and pancreatic MiaPaCa-2 cell lines] [4]

Cytotoxicity of roots crude methanol extract [Significant activity with $I C_{50}$ values of $7.18 ; 7.79$ and $9.17 \mu \mathrm{g} / \mathrm{ml}$ respectively on leukemia CCRFCEM cells, CEM/5000 cells and pancreatic MiaPaCa2 cell lines] [4]

Antimicrobial : Ethanol extract of the seeds on Ca. Cn. Mg. Tm. Tr. Bci. Af. Afl. Sb [6] Essential oil : Sa [15]

Cytotoxicity of seeds crude methanol extract [weak activity on leukemia CCRF-CEM and pancreatic MiaPaCa-2 cell lines and significant activity on CEM/ADR5000 cells with $\mathrm{IC}_{50}$ value of $\left.8.13 \mu \mathrm{g} / \mathrm{ml}\right]$ [4]

Cytotoxicity of roots crude methanol extract [Significant activity with $I C_{50}$ values of 8.4; 7.18 and $12.11 \mu \mathrm{g} / \mathrm{ml}$ respectively on leukemia CCRF-CEM cells, CEM/5000 cells and pancreatic MiaPaCa-2 cell lines] [4]

Fruits, leaves. $\quad$ Antimicrobial : Fm. Afl. Af [18]; Essential oil: Af. BC.

seeds $\quad \overline{B s . ~ C g l . ~ E C . ~ K p . ~ S a . ~ S f ~[15] . ~ C y t o t o x i c i t y ~ o f ~ f r u i t s ~ s e e d s ~}$ methanol extract [weak activity on leukemia CCRFCEM and CEM/ADR5000 cells, and pancreatic MiaPaCa-2 cell lines] [4]

Cytotoxicity of roots crude methanol extract [weak activity on leukemia CCRF-CEM and CEM/ADR5000 cells, and pancreatic MiaPaCa-2 cell lines] [4]

Fruits, leaves. bark Insecticidal : Cs [20] Antimicrobial (Q); Ec. Sa. Bs. Pa. Ca. An $[8,22]$

Antimicrobial : Essential oil of stem bark on Ec, Sa, Bs, Cu [23].

Cytotoxicity of fruits crude methanol extract [weak activity on leukemia CCRF-CEM and CEM/ADR5000 cells, and pancreatic MiaPaCa-2 cell lines] [4] 


\section{Table 1 Spices used in the present study and evidence of their activities (Continued)}

\begin{tabular}{|c|c|c|c|c|}
\hline $\begin{array}{l}\text { Tetrapleura tetraptera } \\
\text { (Schum. \& Thonn) Taub. } \\
\text { (Mimosaceae) }\end{array}$ & $12117 /$ SRFC & $\begin{array}{l}\text { Pain, arthritis, epilepsy, } \\
\text { convulsion, gastric ulcer, } \\
\text { cancer }[4,20]\end{array}$ & Bark, leaves. roots & $\begin{array}{l}\text { Cytotoxicity of fruits crude methanol extract [weak } \\
\text { activity on leukemia CCRF-CEM and CEM/ADR5000 } \\
\text { cells, and pancreatic MiaPaCa-2 cell lines] [4] }\end{array}$ \\
\hline \multicolumn{5}{|c|}{$\begin{array}{l}\text { a }(\mathrm{HNC}): \text { Cameroon National Herbarium; (SRFC): Société des reserves forestières du Cameroun; }{ }^{\mathrm{b}}(/): \text { Not reported. } \\
\text { ' }[\mathrm{Screened} \text { activity: significant }(\mathrm{S}: \mathrm{CMl}<100 \mu \mathrm{g} / \mathrm{ml}) \text {. moderate }(\mathrm{M}: 100<\mathrm{CMl} \leq 625 \mu \mathrm{g} / \mathrm{ml}) \text {. Weak }(\mathrm{W}: \mathrm{CMl}>625 \mu \mathrm{g} / \mathrm{ml}) \mathrm{Q}: \text { Qualitative activity based on the } \\
\text { determination of inhibition zone [15]; Af : Aspergillus fumigatus. Afl : Aspergillus flavus. An: Aspergillus niger. Bc : Bacillus cereus. Bci : Botrytis cinerea. Bs : Bacillis } \\
\text { subtilis. Bt: Botryodiploidia theobromae. Ca : Candida albicans. Cd : Clostridium difficile. Cm : Colletotrichum musae. Cn : Cryptococcus neoformans. Cs: Callosobruchus } \\
\text { subinnotatus. Cu : Candida utilis. Ec : Escherichia coli. Fm :Fusarium moniliforme. Fo :Fusarium oxysporum. Fp : Fusarium proliferatum. Lt : Lasiodiplodia theobromae. } \\
\text { Mg : Microsporum gypseum. Pa: Pseudomonas aeruginosa. Sa : Staphylococcus aureus. Sb: Scopulariopsis brevicaulis. Tm: Trichophyton mentagrophytes. Tr: } \\
\text { Trichophyton rubrum. }\end{array}$} \\
\hline
\end{tabular}

the seeds of Fagara leprieurii, Monodora myristica and Piper guineense, the roots of Dorstenia psilurus, Imperata cylindricum and Pentadiplandra brazzeana and the leaves of Cinnamomum zeylanicum. The plants were identified by Mr. Victor Nana of the National herbarium (Yaoundé, Cameroon) where voucher specimens were deposited under a reference number (Table 1). The extracts were obtained by methanol $(\mathrm{MeOH})$ maceration as previously described [5].

\section{Preliminary phytochemical investigations}

The major secondary metabolites classes were screened according to the common phytochemical methods described by Harborne [24].

\section{Chemicals for antimicrobial assays}

Tetracycline (TET), cefepime (FEP), streptomycin (STR), ciprofloxacin (CIP), norfloxacin (NOR), chloramphenicol $(\mathrm{CHL})$, cloxacillin (CLX), ampicillin (AMP), erythromycin (ERY), kanamycin (KAN) (Sigma-Aldrich, St Quentin Fallavier, France) were used as reference antibiotic. $p$-Iodonitrotetrazolium chloride (INT) and phenylalanine arginine $\beta$-naphthylamide (PAßN) were used as microbial growth indicator and efflux pumps inhibitor (EPI) respectively.

\section{Bacterial strains and culture media}

The studied microorganisms included reference (from the American Type Culture Collection) and clinical (Laboratory collection) strains of Providencia stuartii, Pseudomonas aeruginosa, Klebsiella pneumoniae, Escherichia coli, Enterobacter aerogenes and Enterobacter cloacae The bacterial strains and their features were previously reported [5]. The preliminary treatment of these organisms as well as the culture media were conducted as previously described [5].

\section{Bacterial susceptibility determinations}

The respective MICs of samples on the studied bacteria were determined using rapid INT colorimetric assay $[25,26]$ with some modifications as previously reported [5]. The inoculum concentration used was $1.5 \times 10^{6}$ $\mathrm{CFU} / \mathrm{ml}$ and the samples were incubated at $37{ }^{\circ} \mathrm{C}$ for

Table 2 Extraction yields, aspects and phytochemical composition of the plant extracts

\begin{tabular}{|c|c|c|c|c|c|c|c|c|c|c|c|}
\hline \multirow{3}{*}{$\begin{array}{l}\text { Spice samples } \\
\text { Aframomum citratum }\end{array}$} & \multirow{2}{*}{\multicolumn{2}{|c|}{$\begin{array}{l}\text { Extraction Physical aspect } \\
\text { yield }(\%)^{*}\end{array}$}} & \multicolumn{9}{|c|}{ Phytochemical composition } \\
\hline & & & \multicolumn{3}{|c|}{ Alkaloids Anthocyanins Anthraquinons } & \multicolumn{6}{|c|}{ Flavonoids Phenols Saponins Sterols Tannins Triterpenes } \\
\hline & 2.6 & Oily, dark green & + & - & - & + & + & - & - & + & + \\
\hline Aframomum melegueta & 7.3 & Brown powder & + & - & - & - & - & + & - & - & + \\
\hline $\begin{array}{l}\text { Cinnamomum } \\
\text { zeylanicum }\end{array}$ & 8.4 & Oily, dark green & + & - & - & + & + & - & + & + & - \\
\hline Dorstenia psilurus & 10.3 & Oily, brown & + & + & + & + & + & + & - & + & + \\
\hline Fagara leuprieurii & 26.2 & Creamy, brown & + & - & + & + & + & - & - & + & + \\
\hline Imperata cylindricum & 8.2 & Creamy, brown & + & + & + & + & + & - & - & - & + \\
\hline Monodora myristica & 23.5 & Oily, brown & + & - & + & + & + & - & - & - & + \\
\hline $\begin{array}{l}\text { Pentadiplandra } \\
\text { brazzeana }\end{array}$ & 4.6 & Creamy, brown & + & - & - & + & + & - & - & - & - \\
\hline Piper guineense & 17.5 & Creamy, brown & + & - & + & + & + & - & - & - & - \\
\hline Scorodophloeus zenkeri & 9.2 & $\begin{array}{l}\text { Creamy, dark } \\
\text { green }\end{array}$ & + & - & - & + & + & - & - & + & - \\
\hline Tetrapleura tetraptera & 29.4 & brown & + & - & + & + & + & + & - & + & + \\
\hline
\end{tabular}

(+): Present; (-): Absent; ${ }^{*}$ The yield was calculated as the ratio of the obtained methanol extract according to the initial mass of the spice powder. 
Table 3 Minimal inhibitory concentration (MIC) of the studied plants extracts and chloramphenicol on the studied bacterial species

Bacterial Tested samples and MIC in $\mu \mathrm{g} / \mathrm{ml}$ in the absence and presence of PAßN (in parenthesis)

\begin{tabular}{|c|c|c|c|c|c|c|c|c|c|c|c|c|}
\hline \multirow{2}{*}{ strains } & \\
\hline & $\begin{array}{l}\text { Aframomum } \\
\text { citratum }\end{array}$ & $\begin{array}{l}\text { Aframomum } \\
\text { melegueta }\end{array}$ & $\begin{array}{l}\text { Imperata } \\
\text { cylindricum }\end{array}$ & $\begin{array}{l}\text { Cinnamomum } \\
\text { zeylanicum }\end{array}$ & $\begin{array}{l}\text { Dorstenia } \\
\text { psilurus }\end{array}$ & $\begin{array}{l}\text { Fagara } \\
\text { leprieuri }\end{array}$ & $\begin{array}{l}\text { Monodora } \\
\text { myristica }\end{array}$ & $\begin{array}{l}\text { Pentadiplandra } \\
\text { brazzeana }\end{array}$ & $\begin{array}{l}\text { Piper } \\
\text { guineense }\end{array}$ & $\begin{array}{l}\text { Scorodophloeus } \\
\text { zenkeri }\end{array}$ & $\begin{array}{l}\text { Tetrapleura } \\
\text { tetraptera }\end{array}$ & CHL \\
\hline \multicolumn{13}{|l|}{ E. coli } \\
\hline ATCC8739 & 512 & 512 & 512 & 64 & - & 512 & 1024 & 1024 & 1024 & 1024 & 1024 & 1 \\
\hline ATCC10536 & 1024 & 512 & 1024 & 512 & 128 & 256 & 1024 & 512 & 1024 & 512 & 1024 (1024) & $\begin{array}{l}32 \\
(<2)\end{array}$ \\
\hline AG100 & 1024 (1024) & 1024 (1024) & $1024(256)$ & $-(64)$ & $-(1024)$ & $1024(1024)$ & 512 & 1024 (1024) & $1024(512)$ & 1024 (1024) & 1024 (1024) & $\begin{array}{l}4 \\
(<2)\end{array}$ \\
\hline AG100A & $512(128)$ & 1024 (1024) & $1024(128)$ & $512(128)$ & $512(128)$ & $512(512)$ & 1024 (1024) & $-(-)$ & $1024(1024)$ & 1024 (1024) & 1024 (1024) & $\begin{array}{l}<2 \\
(<2)\end{array}$ \\
\hline AG100A $A_{T E T}$ & $512(512)$ & 1024 (1024) & 1024 (1024) & $512(512)$ & $512(128)$ & $1024(1024)$ & - & - & 1024 & 512 & 1024 & $\begin{array}{l}32 \\
(<2)\end{array}$ \\
\hline AG102 & 1024 & - & 1024 & 1024 & 512 & 1024 & - & - & - & - & - & $\begin{array}{l}16 \\
(<2)\end{array}$ \\
\hline MC4100 & $512(512)$ & $512(256)$ & 1024 (1024) & 1024 (1024) & $512(256)$ & 512 & $-(-)$ & 1024 (1024) & 1024 (1024) & $1024(1024)$ & $512(512)$ & $\begin{array}{l}4 \\
(<2)\end{array}$ \\
\hline W3110 & $512(256)$ & $512(512)$ & $512(512)$ & $512(512)$ & $512(256)$ & 256 & 512 & 1024 (1024) & 1024 (128) & 512 & $512(512)$ & $\begin{array}{l}1 \\
(<2)\end{array}$ \\
\hline \multicolumn{13}{|l|}{ E. aerogenes } \\
\hline ATCC13048 & 1024 & - & 1024 & 1024 & 1024 & 1024 & 1024 & - & - & - & - & $\begin{array}{l}8 \\
(<2)\end{array}$ \\
\hline CM64 & $1024(1024)$ & $1024(1024)$ & $512(128)$ & $1024(512)$ & $512(256)$ & $1024(1024)$ & $1024(1024)$ & 1024 (1024) & 1024 (1024) & $1024(1024)$ & $512(512)$ & 32 \\
\hline EA27 & $512(512)$ & 1024 (1024) & $512(512)$ & $512(512)$ & $-(-)$ & $1024(1024)$ & 1024 (1024) & 1024 (1024) & $-(-)$ & 1024 (1024) & $1024(512)$ & $\begin{array}{l}64 \\
(32)\end{array}$ \\
\hline EA289 & - & 1024 & - & 1024 & - & - & 1024 & 1024 & - & - & 1024 & 256 \\
\hline EA298 & 1024 & 512 & - & - & 1024 & - & 256 & 256 & 512 & 256 & 1024 & 256 \\
\hline EA3 & - & - & - & - & - & 1024 & - & - & - & - & - & 256 \\
\hline \multicolumn{13}{|l|}{ E. cloacae } \\
\hline BM47 & $512(512)$ & 1024 (1024) & 1024 (1024) & $1024(512)$ & $1024(128)$ & $1024(1024)$ & $1024(1024)$ & 1024 & 1024 & 1024 & 1024 & $-(8)$ \\
\hline BM67 & $512(512)$ & 1024 (1024) & 1024 (1024) & 1024 (1024) & $1024(128)$ & $-(-)$ & $-(-)$ & $-(-)$ & $-(-)$ & $-(-)$ & $-(-)$ & $-(32)$ \\
\hline ECCI69 & $512(512)$ & 1024 (1024) & 1024 (1024) & 1024 (1024) & $-(-)$ & $-(-)$ & 1024 (1024) & $-(-)$ & 1024 (1024) & $1024(1024)$ & $1024(512)$ & $-(32)$ \\
\hline \multicolumn{13}{|c|}{ K. pneumoniae } \\
\hline ATCC12296 & 1024 & 1024 & 1024 & 1024 & 1024 & 1024 & 512 & - & - & - & 1024 & 4 \\
\hline K2 & 1024 & - & 1024 & 1024 & 1024 & - & 1024 & - & - & - & - & - \\
\hline K24 & 1024 & 1024 & 1024 & 1024 & 1024 & 1024 & 512 & - & - & 1024 & 1024 & $\begin{array}{l}32 \\
(<2)\end{array}$ \\
\hline
\end{tabular}


Table 3 Minimal inhibitory concentration (MIC) of the studied plants extracts and chloramphenicol on the studied bacterial species (Continued)

\begin{tabular}{|c|c|c|c|c|c|c|c|c|c|c|c|c|}
\hline KP55 & 512 & 1024 & 256 & 512 & 512 & 1024 & 1024 & - & - & - & 1024 & $\begin{array}{l}32 \\
(<2)\end{array}$ \\
\hline KP63 & $512(512)$ & 1024 (1024) & 1024 (1024) & $512(512)$ & $512(128)$ & $1024(512)$ & 1024 (1024) & 512 & 1024( & $1024(512)$ & 1024 (1024) & $\begin{array}{l}64 \\
(<2)\end{array}$ \\
\hline \multicolumn{13}{|l|}{ P. stuartuii } \\
\hline ATCC29916 & $1024(1024)$ & $-(-)$ & $-(-)$ & $-(-)$ & $1024(1024)$ & $-(-)$ & $-(-)$ & $-(-)$ & 1024( & $1024(1024)$ & 1024 (1024) & 8 \\
\hline NEA16 & 1024 (512) & $-(-)$ & 1024 (1024) & $512(512)$ & $512(256)$ & 1024 & 1024 & - & - & 1024 & - & $\begin{array}{l}64 \\
(<2)\end{array}$ \\
\hline PS2636 & 1024 & - & - & - & - & - & - & - & - & - & - & - \\
\hline PS299645 & 512 & 512 & 1024 & 1024 & 1024 & 1024 & - & 1024 & 1024 & 512 & 1024 & 128 \\
\hline \multicolumn{13}{|c|}{ P. aeruginosa } \\
\hline PA01 & - & - & - & - & - & - & - & - & - & - & - & - \\
\hline PA124 & - & - & - & - & - & - & - & - & - & 1024 & - & $\begin{array}{l}32 \\
(<2)\end{array}$ \\
\hline
\end{tabular}

(-): MIC not detected at up to $1024 \mu \mathrm{g} / \mathrm{ml}$ for the les extracts and $256 \mu \mathrm{g} / \mathrm{ml}$ for chloramphenicol. () : values in parenthesis are MIC of substance in the presence of PABN at $20 \mu \mathrm{g} / \mathrm{ml}$. The MIC of PAßN was $64 \mu \mathrm{g} / \mathrm{ml}$

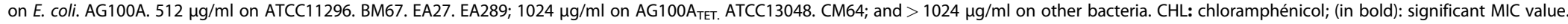


Table 4 Minimal inhibitory concentration (MIC) in $\mu \mathrm{g} / \mathrm{ml}$ of antibiotics in the absence and presence sub-inhibitory concentrations of Aframomum citratum extract against some MDR bacteria

\begin{tabular}{|c|c|c|c|c|c|c|c|c|c|c|c|c|c|c|c|}
\hline \multirow[t]{3}{*}{ Bacterial strains } & \multicolumn{15}{|c|}{ Antibiotics and MIC in absence and presence of Aframomum citratum extract } \\
\hline & \multicolumn{3}{|c|}{ Ampicillin } & \multicolumn{4}{|c|}{ Cefepime } & \multicolumn{3}{|c|}{ Chloramphenicol } & \multicolumn{3}{|c|}{ Ciprofloxacin } & \multirow{2}{*}{\multicolumn{2}{|c|}{ Cloxacillin }} \\
\hline & Alone & $\mathrm{MIC} / 2.5$ & MIC/5 & on & $\mathrm{MIC} / 2.5$ & MIC/5 A & Alone & $\mathrm{MIC} / 2.5$ & $\mathrm{MIC} / 5$ & Alone & $\mathrm{MIC} / 2.5$ & $\mathrm{MIC} / 5$ & Alone & & \\
\hline AG10 & - & - & - & - & - & - & 256 & $32(8)^{5}$ & $64(4)^{5}$ & 256 & $128(2)^{\mathrm{S}}$ & $256(1)^{\prime}$ & - & - & - \\
\hline AG1 & - & - & - & 128 & $28(1)^{\prime}$ & $128(1)^{\prime}$ & 16 & $8(2)^{5}$ & $8(2)^{5}$ & $<2$ & 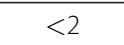 & $<$ & - & - & - \\
\hline CM64 & - & $256(1)^{1}$ & - & - & $64(>4)^{5}$ & - & $\mathrm{nt}$ & 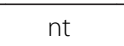 & $\mathrm{ml}$ & nt & 111 & 111 & - & 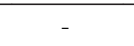 & - \\
\hline KP63 & - & $32(>8)^{5}$ & - & 256 & $32(8)^{5}$ & $256(1)^{\prime}$ & - & $64(>4)^{5} 2$ & $256(>1)^{\mathrm{S}}$ & 64 & $64(1)^{\prime}$ & $64(1)^{1}$ & - & $256(>1)^{\mathrm{S}}$ & - \\
\hline PA124 & 28 & $16(8)^{S}$ & $64(2)^{S}$ & 128 & $128(1)^{\prime} 2$ & $256(0.5)^{\prime}$ & 32 & $16(2)^{S}$ & $16(2)^{S}$ & 16 & $4(4)^{5}$ & $16(1)^{\prime}$ & - & - & - \\
\hline \multirow[t]{2}{*}{ Bacterial strains } & \multicolumn{3}{|c|}{ Erythromycin } & \multicolumn{3}{|c|}{ Kanamycin } & \multicolumn{3}{|c|}{ Norfloxacin } & \multicolumn{3}{|c|}{ Streptomycin } & \multicolumn{3}{|c|}{ Tetracyclin } \\
\hline & Alone & $\mathrm{MIC} / 2.5$ & MIC/5 & Alon & & $\mathrm{MIC} / 5$ & Alone & & MIC/5 & Al & & $\mathrm{MIC} / 5$ & Alone & & ind \\
\hline AG100Atet & 64 & $16(4)^{S}$ & $32(2)^{5}$ & - & $32(>8)^{5}$ & 256 & 128 & $16(8)^{S}$ & $128(1)^{\prime}$ & $<2$ & $<2$ & $<2$ & 2 & $<2(>1)^{\mathrm{S}}$ & $2(1)^{1}$ \\
\hline AG102 & 32 & $16(2)^{S}$ & $16(2)^{S}$ & $<2$ & $<2$ & $<2$ & $<2$ & $<2$ & $<2$ & - & $128(>2)^{\mathrm{S}}$ & $256(>1)^{5}$ & $<<2$ & $<2$ & $<2$ \\
\hline CM64 & - & $128(>2)^{\mathrm{S}} 2$ & $256(>1)^{\mathrm{S}}$ & 4 & $<2$ & $<2$ & 4 & $<2(>2)^{5}$ & $4(1)^{\prime}$ & 32 & $4(8)^{5}$ & $8(4)^{5}$ & nt & nt & $\mathrm{nt}$ \\
\hline KP63 & 16 & $<1(>16)^{\mathrm{S}}$ & $4(4)^{5}$ & 32 & $16(2)^{S}$ & $32(1)^{1}$ & - & $128(>2)^{\mathrm{S}}$ & $256(>1)^{S}$ & $<4$ & $<4$ & $<4$ & $<2$ & $<2$ & $<2$ \\
\hline PA124 & 128 & $64(2)^{s}$ & $64(2)^{\mathrm{s}}$ & 128 & $16(8)^{\mathrm{s}}$ & $64(2)^{s}$ & 64 & $8(8)^{5}$ & $32(2)^{s}$ & $\mathrm{nt}$ & $\mathrm{nt}$ & $\mathrm{nt}$ & 8 & $2(4)^{S} 2$ & $2(4)^{5}$ \\
\hline
\end{tabular}

MIC/2.5: concentration of plant extract added equal to $204.8 \mu \mathrm{g} / \mathrm{mL}$ for AG100A MIC/5: concentration of plant extract added equal to $102.4 \mu \mathrm{g} / \mathrm{mL}$ for AG100A (): Folds decreasing of MIC. S: synergy. I: indifference. nt: not tested; (-): MIC $>256$.

$18 \mathrm{~h}$ [5]. The final concentration of DMSO was lower than $2.5 \%$ and this concentration also served as negative control [5]. Chloramphenicol was used as reference antibiotic. The MICs of samples were detected after $18 \mathrm{~h}$ incubation at $37^{\circ} \mathrm{C}$, following addition $(40 \mu \mathrm{l})$ of $0.2 \mathrm{mg} / \mathrm{ml}$ INT and incubation at $37^{\circ} \mathrm{C}$ for 30 minutes [5]. MIC was defined as the lowest sample concentration that prevented the color change of the medium and exhibited complete inhibition of microbial growth [27].

Samples were tested alone and then, in the presence of $\mathrm{PA} ß \mathrm{~N}$ at $20 \mathrm{mg} / \mathrm{L}$ final concentration as previously reported [5]. Four of the best extracts, those from $A$. citratum, C. zeylanicum, D. psilurus and T. tetraptera were also tested in association [5] at the concentrations selected following a preliminary assay on $P$. aeruginosa PA124 (See Additional file 1: Table S1). All assays were performed in triplicate and repeated thrice. Fractional inhibitory concentration (FIC) [5] were calculated and the interpretations were made as follows: synergistic $(<0.5)$, indifferent $(0.5$ to 4$)$, or antagonistic $(>4)$ [28] (The FIC values available in Additional file 1: Table S2 and S3).

Table 5 Minimal inhibitory concentration (MIC) of antibiotics in absence and presence of Cinnamomum zeylanicum extract $(\mu \mathrm{g} / \mathrm{mL})$

\begin{tabular}{|c|c|c|c|c|c|c|c|c|c|c|c|c|c|c|c|}
\hline \multirow[t]{3}{*}{ Bacterial strains } & \multicolumn{15}{|c|}{ Antibiotics and MIC in absence and presence of Cinnamomum zeylanicum extract } \\
\hline & \multicolumn{3}{|c|}{ Ampicillin } & \multicolumn{3}{|c|}{ Cefepime } & \multicolumn{3}{|c|}{ Chloramphenicol } & \multicolumn{3}{|c|}{ Ciprofloxacin } & \multicolumn{3}{|c|}{ Cloxacillin } \\
\hline & lone & & $\mathrm{MIC} / 5$ & Alone & MIC & MIC/5 A & Alone & & & Alone & & $\mathrm{MIC} / 5$ & Alone & r & $\mathrm{MIC} / 5$ \\
\hline AG10 & - & - & - & - & - & - & 256 & $16(16)^{\mathrm{s}}$ & $32(8)^{5}$ & 256 & $64(4)^{5}$ & $128(2)^{\mathrm{S}}$ & - & - & - \\
\hline AG1 & - & - & - & 128 & 256 & $256(1)^{\prime}$ & 16 & $8(2)^{5}$ & $16(1)^{\prime}$ & $<2$ & $<2$ & $<2$ & - & $256(>1)^{\mathrm{S}}$ & - \\
\hline CM64 & - & $256(>1)$ & - & -2 & $256(>1)^{\mathrm{S}}$ & - & nt & nt & nt & nt & nt & $\mathrm{nt}$ & - & - & - \\
\hline KP63 & - & $32(>8)^{5}$ & - & 256 & $32(8)^{\mathrm{s}}$ & $256(1)^{\prime}$ & - & $32(>8)^{\mathrm{s}}$ & $256(>1)^{S}$ & 64 & $128(0.5)^{\prime}$ & $128(0.5)^{1}$ & - & $64(>4)^{5}$ & $56(>$ \\
\hline PA124 & 28 & $16(8)^{S}$ & $64(2)^{S}$ & 28 & $128(1)^{\prime}$ & $128(1)^{\prime}$ & 32 & $2(16)^{s}$ & $8(4)^{5}$ & 16 & $8(2)^{5}$ & $16(1)^{\prime}$ & - & - & - \\
\hline \multirow[t]{2}{*}{ Bacterial strains } & \multicolumn{3}{|c|}{ Erythromycin } & \multicolumn{3}{|c|}{ Kanamycin } & \multicolumn{3}{|c|}{ Norfloxacin } & \multicolumn{3}{|c|}{ Streptomycin } & \multicolumn{3}{|c|}{ Tetracycline } \\
\hline & Alo & $\mathrm{MIC} / 2.5$ & MIC/5 & Alone & $M I C / 2.5$ & MIC/5 A & Alone & $\mathrm{MIC} / 2.5$ & MIC/5 & Alone & $\mathrm{MIC} / 2.5$ & $\mathrm{MIC} / 5$ & A & & $\mathrm{MIC} / 5$ \\
\hline AG100Atet & 64 & $16(4)^{S}$ & $32(2)^{S}$ & - & $16(>16)^{S} 1$ & $128(>2)^{5}$ & 128 & $128(1)^{\prime}$ & $128(1)^{\prime}$ & $<2$ & $<2$ & $<2$ & 2 & $2(1)^{\prime}$ & $2(1)^{\prime}$ \\
\hline AG102 & 32 & $16(2)^{\mathrm{S}}$ & $16(2)^{S}$ & $<2$ & $<2$ & $<2$ & $<2$ & $<2$ & $<2$ & -2 & $256(>1)^{s}$ & $256(>1)^{\mathrm{S}}$ & $s<2$ & $<2$ & $<2$ \\
\hline CM64 & - & $128(>2)^{\mathrm{S}} 2$ & $256(>1)^{\mathrm{S}}$ & 4 & $<2(>2)^{s}$ & $<2(>2)^{\mathrm{s}}$ & 54 & $<2(>2)^{\mathrm{s}}$ & $4(1)^{\prime}$ & 32 & $4(8)^{5}$ & $8(4)^{5}$ & nt & $\mathrm{nt}$ & $\mathrm{nt}$ \\
\hline KP63 & 16 & $1(16)^{\mathrm{s}}$ & $4(4)^{5}$ & 32 & $32(1)^{\prime}$ & $32(1)^{\prime}$ & - & $128(>2)^{\mathrm{S}}$ & $256(>1)^{S}$ & $<4$ & $<4$ & $<4$ & $<2$ & $<2$ & $<2$ \\
\hline PA124 & 128 & $16(8)^{S}$ & $32(4)^{s}$ & 128 & $8(16)^{5}$ & $32(4)^{s}$ & 64 & $32(2)^{5}$ & $64(1)^{\prime}$ & $\mathrm{nt}$ & nt & $\mathrm{nt}$ & 8 & $2(4)^{s}$ & $2(4)^{5}$ \\
\hline
\end{tabular}

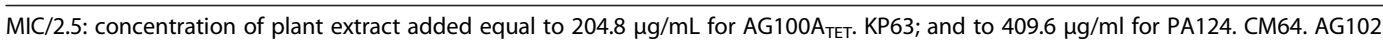

MIC/5: concentration of plant extract added equal to $102.4 \mu \mathrm{g} / \mathrm{mL}$ for AG100A

(): Folds decreasing of MIC. S: synergy. I: indifference. nt: not tested; (-): MIC $>256 \mu \mathrm{g} / \mathrm{ml}$. 
Table 6 Minimal inhibitory concentration (MIC) of antibiotics in absence and presence extracts Dorstenia psilurus ( $\mu \mathrm{g} / \mathrm{ml})$ Bacterial strains Antibiotics and MIC in absence and presence of Dorstenia psilurus extract

\begin{tabular}{|c|c|c|c|c|c|c|c|c|c|c|c|c|c|c|c|}
\hline & \multicolumn{3}{|c|}{ Ampicillin } & \multicolumn{3}{|c|}{ Cefepime } & \multicolumn{3}{|c|}{ Chloramphenicol } & \multicolumn{3}{|c|}{ Ciprofloxacin } & \multicolumn{3}{|c|}{ Cloxacillin } \\
\hline & Ione & e $M I C / 2.5$ & $\mathrm{MIC} / 5$ & Alone & e $M I C / 2$ & MIC/5 & Alone & $\mathrm{MIC} / 2.5$ & $\mathrm{MIC} / 5$ & Alone & $\mathrm{MIC} / 2.5$ & $\mathrm{MIC} / 5$ & Alone & $M I C / 2.5$ & $\mathrm{MIC/5}$ \\
\hline & - & - & - & - & - & - & 256 & $128(2)^{\mathrm{S}}$ & $256(1)^{1}$ & 256 & $64(4)^{5}$ & $128(2)^{\mathrm{S}}$ & - & - & - \\
\hline$A G 1$ & - & - & - & 128 & $256(0$ & $256(0.5)^{\prime}$ & 16 & $4(4)^{5}$ & $4(4)^{5}$ & $<2$ & $<2$ & -2 & 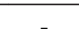 & - & - \\
\hline CM64 & - & $256(>1)^{\mathrm{S}}$ & - & - & $64(>)$ & $>4)^{5}$ & $\mathrm{nt}$ & $\mathrm{nt}$ & $\mathrm{nt}$ & $\mathrm{nt}$ & $\mathrm{nt}$ & $\mathrm{nt}$ & - & $256(>1)^{\mathrm{S}}$ & - \\
\hline KP63 & - & $32(>8)^{5}$ & - & 256 & $64(4)^{5}$ & $128(2)^{\mathrm{S}}$ & - & $64(>4)^{s_{2}}$ & $256(>1)^{S}$ & 564 & $64(1)^{\prime}$ & $64(1)^{\prime}$ & - & $64(>4)^{s}$ & $6(>1$ \\
\hline PA124 & 28 & $64(2)^{\mathrm{S}}$ & $64(2)^{s}$ & 128 & $128(1)^{\prime}$ & $128(1)^{\prime}$ & 32 & $16(2)^{S}$ & $32(1)^{\prime}$ & 16 & $16(1)^{\prime}$ & $16(1)^{\prime}$ & - & 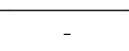 & - \\
\hline \multirow[t]{2}{*}{ Bacterial strain } & \multicolumn{3}{|c|}{ Erythromycin } & \multicolumn{3}{|c|}{ Kanamycin } & \multicolumn{3}{|c|}{ Norfloxacin } & \multicolumn{3}{|c|}{ Streptomycin } & \multicolumn{3}{|c|}{ Tetracycline } \\
\hline & Alone & e $M I C / 2.5$ & $\mathrm{MIC} / 5$ & Alone & $\mathrm{MIC} / 2.5$ & MIC/5 & Alone & $M I C / 2.5$ & MIC/5 & Alone & MIC/2.5 & MIC/5 & Alone & $M I C / 2.5$ & MIC/5 \\
\hline AG100Atet & 64 & $32(2)^{s}$ & $32(2)^{\mathrm{S}}$ & - & $128(>2)^{5}$ & $256(>1)^{s}$ & S 128 & $128(1)^{\prime}$ & $256(0.5)^{\prime}$ & $<2$ & $<2$ & $<2$ & 2 & $2(1)^{1}$ & $2(1)^{\prime}$ \\
\hline AG102 & 32 & $64(0.5)^{\prime}$ & $64(0.5)^{\prime}$ & $<2$ & $<2$ & $<2$ & $<2$ & $<2$ & $<2$ & - & $256(>1)^{S}$ & $256(>1)^{S}$ & $s<2$ & $<2$ & $<2$ \\
\hline CM64 & - & $64(>4)^{s}$ & $256(>1)^{\mathrm{S}}$ & S 4 & $4(1)^{\prime}$ & $8(0.5)^{1}$ & 4 & $<2(>2)^{\mathrm{S}}$ & $2(>2)^{5}$ & 32 & $8(4)^{5}$ & $32(1)^{\prime}$ & $\mathrm{nt}$ & $\mathrm{nt}$ & $\mathrm{nt}$ \\
\hline KP63 & 16 & $1(16)^{s}$ & $8(2)^{\mathrm{s}}$ & 32 & $32(1)^{\prime}$ & $64(0.5)^{\prime}$ & - & - & - & $<4$ & $<4$ & $<4$ & $<2$ & $<2$ & $<2$ \\
\hline PA124 & 128 & $64(2)^{S}$ & $128(1)^{\prime}$ & 128 & $4(32)^{5}$ & $16(8)^{\mathrm{s}}$ & 64 & $32(2)^{S}$ & $64(1)^{\prime}$ & $\mathrm{nt}$ & nt & $\mathrm{nt}$ & 8 & $2(4)^{s}$ & $8(1)^{\prime}$ \\
\hline
\end{tabular}

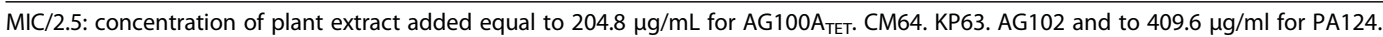

MIC/5: concentration of plant extract added equal to $102.4 \mu \mathrm{g} / \mathrm{mL}$ for AG100A

(): Folds decreasing of MIC. S: synergy. I: indifference. nt: not tested; (-): MIC $>256 \mu \mathrm{g} / \mathrm{ml}$.

\section{Results}

Phytochemical composition of the spice extracts

The results of qualitative analysis showed that each plant contains various phytochemicals compounds such as alkaloids, anthocyanins, anthraquinones, flavonoids, phenols, saponins, steroids, tannins and triterpenes as shown in Table 2.
Antibacterial activity of the spice extracts

The results summarized in Table 3 summarize the MIC of the extract tested alone or in combination with PA $\mathrm{N}$ on the tested microorganisms. Its shows that all the studied extracts were active on at least one microbial strain. $A$. citratum showed the best activity, it inhibitory effect being recorded on $85 \%(24 / 28)$ of the tested bacteria. Other

Table 7 Minimal inhibitory concentration (MIC) of antibiotics in absence and presence extracts Tetrapleura tetraptera $(\mu \mathrm{g} / \mathrm{ml})$

\begin{tabular}{|c|c|c|c|c|c|c|c|c|c|c|c|c|c|c|c|}
\hline \multirow{3}{*}{$\begin{array}{l}\text { Bacterial } \\
\text { strains }\end{array}$} & \multicolumn{15}{|c|}{ Antibiotics and MIC in absence and presence Tetrapleura tetraptera extract } \\
\hline & \multicolumn{3}{|c|}{ Ampicillin } & \multicolumn{3}{|c|}{ Cefepime } & \multicolumn{3}{|c|}{ Chloramphenicol } & \multicolumn{3}{|c|}{ Ciprofloxacin } & \multicolumn{3}{|c|}{ Cloxacillin } \\
\hline & Alon & e $M I C / 2.5$ & $\mathrm{MIC/5}$ & Alone & $\mathrm{MIC} / 2$ & MIC/5 & Alone & $\mathrm{MIC} / 2.5$ & $\mathrm{MIC/5}$ & Alone & $\mathrm{MIC} / 2.5$ & $\mathrm{MIC/5}$ & Alone & $\mathrm{MIC} / 2.5$ & $\mathrm{MIC/5}$ \\
\hline AG100Atet & - & - & - & - & - & - & 256 & $256(1)^{\prime}$ & - & 256 & $128(2)^{s}$ & $128(2)^{\mathrm{S}}$ & - & - & - \\
\hline AG102 & - & - & - & 128 & $256(0.5)^{1}$ & $256(0.5)^{\prime}$ & ${ }^{\mathrm{I}} 16$ & $8(2)^{5}$ & $8(2)^{s}$ & $<2$ & $<2$ & $<2$ & - & - & - \\
\hline CM64 & - & - & - & - & - & - & $\mathrm{nt}$ & $\mathrm{nt}$ & $\mathrm{nt}$ & $\mathrm{nt}$ & $\mathrm{nt}$ & $\mathrm{nt}$ & - & - & - \\
\hline KP63 & - & - & - & 256 & $256(1)^{\prime}$ & - & - & $256(>1)$ & ${ }_{\mathrm{S}} 56(>1)$ & 64 & $64(1)^{\prime}$ & $64(1)^{1}$ & - & ${\underset{s}{S}}_{128}(>2)$ & ${ }_{\mathrm{S}} 56(>1)$ \\
\hline PA124 & 128 & $64(2)^{S}$ & $128(1)^{\prime}$ & 128 & $128(1)^{\prime}$ & $128(1)^{\prime}$ & 32 & $4(8)^{S}$ & $8(4)^{s}$ & 16 & $16(1)^{\prime}$ & $16(1)^{\prime}$ & - & - & - \\
\hline \multirow{2}{*}{$\begin{array}{l}\text { Bacterial } \\
\text { strains }\end{array}$} & \multicolumn{3}{|c|}{ Erythromycin } & \multicolumn{3}{|c|}{ Kanamycin } & \multicolumn{3}{|c|}{ Norfloxacin } & \multicolumn{3}{|c|}{ Streptomycin } & \multicolumn{3}{|c|}{ Tetracycline } \\
\hline & Alon & e $M I C / 2.5$ & $\mathrm{MIC/5}$ & Alone & $M I C / 2.5$ & MIC/5 & Alone & $\mathrm{MIC} / 2.5$ & $\mathrm{MIC/5}$ & Alone & $\mathrm{MIC} / 2.5$ & $\mathrm{MIC/5}$ & Alone & $\mathrm{MIC} / 2.5$ & MIC/5 \\
\hline AG100Atet & 64 & $64(1)^{\prime}$ & $64(1)^{\prime}$ & - & $\underset{\mathrm{S}}{256}(>1)$ & ${ }_{\mathrm{S}}^{256}(>1)$ & 128 & $128(1)^{\prime}$ & $256(0.5)$ & ${ }^{1}<2$ & $<2$ & $<2$ & 2 & $2(1)^{\prime}$ & $2(1)^{\prime}$ \\
\hline AG102 & 32 & $64(0.5)^{\prime}$ & $64(0.5)$ & $<2$ & $<2$ & $<2$ & $<2$ & $<2$ & $<2$ & - & $256(>1)$ & ${ }_{S} 56(>1)$ & $<2$ & $<2$ & $<2$ \\
\hline CM64 & - & $256(>1)^{S}$ & & 4 & $4(1)^{\prime}$ & $8(0.5)^{\prime}$ & 4 & $4(1)^{\prime}$ & $8(0.5)^{\prime}$ & 32 & $16(2)^{\mathrm{s}}$ & $32(1)^{\prime}$ & $\mathrm{nt}$ & $\mathrm{nt}$ & $\mathrm{nt}$ \\
\hline KP63 & 16 & ${ }_{\mathrm{s}}<1(>16)$ & $8(2)^{s}$ & 32 & $32(1)^{\prime}$ & $64(0.5)^{\prime}$ & - & ${ }_{\mathrm{S}} 56(>1)$ & - & $<4$ & $<4$ & $<4$ & $<2$ & $<2$ & $<2$ \\
\hline PA124 & 128 & $64(2)^{s}$ & $64(2)^{s}$ & 128 & $64(2)^{s}$ & $64(2)^{s}$ & 64 & $32(2)^{5}$ & $64(1)^{\prime}$ & $\mathrm{nt}$ & $\mathrm{nt}$ & $\mathrm{nt}$ & 8 & $2(4)^{s}$ & $2(4)^{s}$ \\
\hline
\end{tabular}

$\mathrm{MIC} / 2.5$ : concentration of plant extract added equal to $204.8 \mu \mathrm{g} / \mathrm{mL}$ for CM64 and to $409.6 \mu \mathrm{g} / \mathrm{ml}$ for AG100A

$\mathrm{MIC} / 5$ : concentration of plant extract added equal to $102.4 \mu \mathrm{g} / \mathrm{mL}$ for CM64; and to $204.8 \mu \mathrm{g} / \mathrm{ml}$ for AG100A

(): Folds decreasing of MIC. S: synergy. I: indifference. nt: not tested; (-): MIC $>256 \mu \mathrm{g} / \mathrm{ml}$. 
samples were less active, their inhibitory potencies being observed on $75 \%$ of tested bacteria $(21 / 28)$ for $I$. cylindricum and C. zeylanicum, $67.9 \%$ (19/28) for A. melegueta, D. psilurus, F. leprieuri and T. tetraptera; 64.3\% (18/28) for $M$. myristica and S. zenkeri; $50 \%$ (14/28) for P. guineense and $42.9 \%(12 / 28)$ for P. brazzeana.

\section{Role of efflux pumps in susceptibility of gram negative bacteria to the tested spice extracts}

Potentiating effect of EPI was not observed on tested bacteria when associated with $M$. myristica, P. brazzeana, $T$. tetraptera and S. zenkeri. PA $\beta \mathrm{N}$ weakly increased the activity of A. citratum, A. melegueta, F. leprieuri, I. cylindricum, C. zeylanicum and P. guineense. The activity of D. psilurus in the presence of EPI significantly increased on most of the tested bacteria (except against P. stuartii ATCC29916, E. cloacae ECCI69 and E. aerogenes EA27) (see Table 3).

\section{Effects of the association of some spice extracts with antibiotics}

A. citratum, C. zeylanicum, D. psilurus and T. tetraptera (Tables 4, 5, 6 and 7) were associated to antibiotics in view of evaluating the possible synergistic effect of these associations. A preliminary study using $P$. aeruginosa PA124 was carried out with ten antibiotics (CLX, AMP, ERY, KAN, CHL, TET, FEP, STR, CIP and NOR) to select the appropriate sub-inhibitory concentrations to be used. MIC/2.5 and $\mathrm{MIC} / 5$ were then selected as the sub-inhibitory concentrations (see Additional file 1: Table S1). All of these four extracts were then tested in association with antibiotics previously listed on strains of E. coli AG100A TET $_{\text {and AG102, }}$ E. aerogenes CM64, K. pneumonia KP63 and P. aeruginosa PA124. No antagonistic effect (FIC $>4$ ) was observed between extracts and antibiotics meanwhile indifference was observe between $T$. tetraptera and antibiotics in most of the case (see Tables 5, 6, and 7, Additional file 1: S2, S3, S4 and S5). Significant increase of the activity was observed with the association of the extracts of $A$. citratum and $D$. psilurus on E. aerogenes CM64 and K. pneumoniae KP63, and with $C$. zeylanicum against $K$. pneumoniae KP63. A significant decrease (synergy effect) of MIC values was also observed when ERY was associated with various extracts, and when extracts of $A$. citratum and C. zeylanicum were each combined with aminoglycosides (KAN, STR), the best activity being noted against E. aerogenes CM64.

\section{Discussion}

\section{Phytochemical composition of the spice extracts}

The phytochemical studies revealed the presence of secondary metabolite such as alkaloids, anthocyanins, anthraquinones, flavonoids, phenols, saponins, sterols, tannins and triterpenes; several molecules belonging to these classes of secondary metabolites were found active on pathogenic microorganisms [29].

\section{Antibacterial activity of the spice extract}

Although this is the first time that plants used in this work are studied for their activities vis-à-vis multiresistant bacteria, plants belonging to some of the genus studied herein, like the Aframomum genus are well documented for their antimicrobial activity [6]. Some antibacterial compounds, such as acridone and chelerythrine have previously been isolated from the fruits of F. leprieurii $[14,30]$. The antimicrobial activity of $P$. brazzeana and S. zenkeri is mainly due to some sulfur compounds. In fact, sulfur compounds with antimicrobial properties have previously been isolated from the two plants $[7,31]$. Several alkaloids of the genus Piper proved to be responsible for the activity of $P$. guineense [32]. The detection of this class of secondary metabolites in the extract studied herein can explain the observed activities. According to Krishnaiah et al. [16], the antimicrobial activity of $I$. cylindricum can be due to the presence of tannins in this plant. However, tannins were not detected in the extract of $I$. cylindricum as found in the present work (Table 2), suggesting that other classes of secondary metabolites might be responsible for the antibacterial activity of this plant.

\section{Role of efflux pumps in susceptibility of gram negative bacteria to the tested spice extracts}

The significant increase of the activity of the extract of $D$. psilurus in the presence of EPI, indicates that bioactive constituents of this plant extract are substrate of efflux pumps. Efflux through AcrAB-TolC pumps was reported as essential mode of resistance of several Gram-negative MDR bacteria to a number of flavonoids isolated from plants of the genus Dorstenia, such as isobavachalcone, kanzonol C, stipulin, etc. [4,15,33-35]. This suggests that possible combination of the extract of $D$. psilurus with EPI can be envisaged to overcome MDR bacteria.

\section{Effects of the association of extracts with antibiotics}

The results obtained by combining the antibiotic with the extracts of $A$. citratum, C. zeylanicum, D. psilurus and $T$. tetraptera indicate that these extracts contain chemical compounds that can modulate the activity of antibiotics against bacteria expressing MDR phenotypes. The methanol extracts of A. citratum, C. zeylanicum and $D$. psilurus showed a synergistic effect with antibiotics inhibiting bacterial cell wall synthesis (AMP and CEF) on K. pneumoniae KP63. The intrinsic mode of action of the active extracts is to be investigated.

\section{Conclusion}

The present work evidently provides information in the role of some Cameroonian spices in the fight against multi-resistant bacteria. The study also highlights the potential of $D$. psilurus as a strong antibacterial agent 
when the extract is combined with efflux pump inhibitor and several antibiotics.

\section{Additional file}

\begin{abstract}
Additional file 1: Table S1. Activities of antibiotics in combination with the sub-inhibitory concentrations of some plants extracts on Pseudomonas aeruginosa PA124. S2. Fractional inhibitory Concentrations of the association between antibiotics and extracts of Aframomum citratum at $\mathrm{MIC} / 2.5$ and $\mathrm{MIC} / 5(\mu \mathrm{g} / \mathrm{ml})$ against MDR bacteria. S3. Fractional inhibitory Concentrations of the association between antibiotics and extracts of Cinnamomum zeylanicum at MIC/2.5 and MIC/5 $(\mu \mathrm{g} / \mathrm{ml})$ against MDR bacteria. S4. Fractional inhibitory Concentrations of the association between antibiotics and extracts of Dorstenia psilurus at $\mathrm{MIC} / 2.5$ and MIC/5 $(\mu \mathrm{g} / \mathrm{ml})$ against MDR bacteria. S5. Fractional inhibitory Concentrations of the association between antibiotics and extracts of Tetrapleura tetraptera at MIC/2.5 and MIC/5 $(\mu \mathrm{g} / \mathrm{ml})$ against MDR bacteria.
\end{abstract}

\section{Competing interest}

The authors declare that they have no competing interest.

\section{Authors' contributions}

IKV carried out the study; VK designed the experiments and wrote the manuscript; VK, GAF, JAKN, JPD, JRK and JMP supervised the work; VK and JMP provided the bacterial strains; All authors read and approved the final manuscript.

\section{Acknowledgements}

Authors are thankful to the Cameroon National Herbarium (Yaounde) for plants identification, Mr Simplice R. Mouokeu for its technical support, and Mr. Paul K. Lunga for language editing.

\section{Author details}

${ }^{1}$ Department of Biochemistry, Faculty of science, University of Dschang, P.O. Box 67, Dschang, Cameroon, Africa. ${ }^{2}$ Transporteurs Membranaires, Chimiorésistance et Drug Design, UMR-MD1, IFR 88, UFRs de Médecine et de Pharmacie, Marseille, France.

\section{Received: 27 January 2012 Accepted: 4 June 2012}

Published: 15 June 2012

\section{References}

1. Ziegler J: L'Empire de la honte. Paris, France: Ed Fayard; 2005

2. OCDE: Partenariat contre les maladies infectieuses. 2009 2009. http://www. observateurocde.org/news/fullstory.php/aid/743/

Partenariat_contre_les_maladies_infectieuses.html, 2009. Accessed on 22 February 2010

3. Yala D, Merad AS, Mohamedi D, Ouar Korich MN: Classification et mode d'action des antibiotiques. Médecine du Maghreb 2001, 91:5-12.

4. Kuete V, Krusche B, Youns M, Voukeng I, Fankam AG, Tankeo S, Lacmata S, Efferth T: Cytotoxicity of some Cameroonian spices and selected medicinal plant extracts. J Ethnopharmaco/ 2011, 134(3):803-812.

5. Fankam AG, Kuete V, Voukeng IK, Kuiate JR, Pages JM: Antibacterial activities of selected Cameroonian spices and their synergistic effects with antibiotics against multidrug-resistant phenotypes. BMC Complement Altern Med. 2011, 11:104

6. Titanji VPK, Zofou D, Ngemenya MN: The antimalarial potential of medicinal plants used for the treatment of malaria in Cameroonian folk medicine. Afr J Trad CAM 2008, 5(3):302-321.

7. Ngemenya MN, Mbah JA, Tane P, Titanji VPK: Antibacterial effects of some Cameroonian medicinal plants against common pathogenic bacteria. Afr J Trad CAM 2006, 3(2):84-93.

8. Konning $\mathrm{GH}$, Agyare C, Ennison B: Antimicrobial activity of some medicinal plants from Ghana. Fitoterapia 2004, 75(1):65-67.

9. Okigbo RN, Ogbonnaya UO: Antifungal effects of two tropical plant leaf extracts (Ocimum gratissimum and Aframomum melegueta) on postharvest yam (Dioscorea spp.) rot. Afr J Biotechnol 2006, 5(9):727-731.
10. Ranasinghe $L$, Jayawardena $B$, Abeywickrama $K$ : Fungicidal activity of essential oils of Cinnamomum zeylanicum (L.) and Syzygium aromaticum (L.) Merret L. M. Perry against crown rot and anthracnose pathogens isolated from banana. Lett Appl Microbiol 2002, 35(3):208-211.

11. Shahverdi AR, Monsef-Esfahani HR, Tavasoli F, Zaheri A, Mirjani R: Trans-Cinnamaldehyde from Cinnamomum zeylanicum bark essential oil reduces the Clindamycin resistance of Clostridium difficile in vitro. $J$ Food Sci 2007, 72(1):55-58.

12. Abegaz BM, Ngadjui BT, Dongo E, Bezabiha MT: Chemistry of the Genus Dorstenia. Curr Org Chem 2000, 4(10):1079-1090.

13. Dimo T, Rakotonirina A, Tan PV, Dongo E, Dongmo AB, Kamtchouing P, Azay J, Abegaz BM, Cros G, Ngadjui TB: Antihypertensive effects of Dorstenia psilurus extract in fructose-fed hyperinsulinemic, hypertensive rats. Phytomedicine 2001, 8(2):101-106.

14. Ngono AN, Biyiti L, Amvam Zollo PH, Bouchet P: Evaluation of antifungal activity of extracts of two Cameroonian Rutaceae: Zanthoxylum leprieurii Guill. etPerr., and Zanthoxylum xanthoxyloides Waterm. J Ethnopharmacol 2000, 70(3):335-342.

15. Kuete V: Potential of Cameroonian plants and derived products against microbial infections: a review. Planta Med 2010, 76(14):1479-1491.

16. Krishnaiah D, Devi T, Bono A, Sarbatly R: Studies on phytochemical constituents of six Malaysian medicinal plants. J Med Plant Res 2009, 3(2):67-72.

17. Nishimoto $K$, Ito $M$, Natori S, Ohmoto T: The structures of arundoin, cylindrin and fernenoltriterpenoids of fernane and arborane of Imperata cylindrica var. koenigif. Terrahdron 1968, 24:735-752

18. Nguefack J, Letha V, Amvam Zollo PH, Mathur SB: Evaluation of five essential oils from aromatic plants of Cameroon for controlling food spoilage and mycotoxin producing fungi. Int J Food Microbiol 2004, 94(3):329-334

19. Burubai W, Akor AJ, Igoni AH, Puyate YT: Some physical properties of African nutmeg (Monodora myristica). Int Agrophysics 2007, 21:123-126.

20. Noumi E, Dibakto TW: Medicinal plants used for peptic ulcer in the Bangangte region, western Cameroon. Fitoterapia 2000, 71(4):406-412.

21. Oparaeke AM, Bunmi JO: Bioactivity of two powdered spices Piper guineense thonn \& schum and Xylopia aethiopica (dunal) a. Richard] as home-made insecticides against Callosobruchus subinnotatus (pic.) on stored bambarra groundnut. Agric Trop Subtrop 2006, 39(2):132-134.

22. Nwinyi OC, Chinedu NS, Ajani OO, Ikpo CO, Ogunniran KO: Antibacterial effects of extracts of Ocimum gratissimum and Piper guineense on Escherichia coli and Staphylococcus aureus. Afr J Food Sci 2009, 3(3):77-81.

23. Kouokam JC, Jahns T, Becker H: Antimicrobial Activity of the Essential Oil and Some isolated Sulfur-Rich Compounds from Scorodophloeus zenkeri. Planta Med 2002, 68(12):1082-1087.

24. Harborne JB: Phytochemical Methods. New York, USA: Chapman and Hall; 1973.

25. Eloff JN: A sensitive and quick microplate method to determine the minimal inhibitory concentration of plant extracts for bacteria. Planta Med 1998, 64(8):711-713.

26. Mativandlela SPN, Lall N, Meyer JJM: Antibacterial, antifungal and antitubercular activity of (the roots of) Pelargonium reniforme (CURT) and Pelargonium sidoides (DC) (Geraniaceae) root. S Afr J Bot 2006, 72(2): 232-237.

27. Kuete V, Ngameni B, Fotso Simo CC, Kengap Tankeu R, Tchaleu Ngadjui B, Meyer JJM, Lall N, Kuiate JR: Antimicrobial activity of the crude extracts and compounds from Ficus chlamydocarpa and Ficus cordata (Moraceae). J Ethnopharmacol 2008, 120(1):17-24.

28. Coutinho HD, Vasconcellos A, Freire-Pessôa HL, Gadelha CA, Gadelha TS, Almeida-Filho GG: Natural products from the termite Nasutitermes corniger lower aminoglycoside minimum inhibitory concentrations. Pharmacogn Mag 2010, 6(21):1-4.

29. Cowan MM: Plant products as antimicrobial agents. Clin Microbio/ Rev 1999, 12(4):564-582.

30. Adesina SK: The Nigerian Zanthoxylum; chemical and biological values. Afr J Trad CAM 2005, 2(3):282-301.

31. El Migirab S, Bergbr Y, Jadot J: Isothiocyanates, Thiourees et Thiocarbamates isolés de Pentadiplandra brazzeana. Phytochemistry 1977 16(11):1719-1721.

32. Parmar VS, Jain SC, Bisht KS, Jain R, Taneja $P$, Jha A, Tyagi OD, Prasad AK, Wengel J, Olsen CE, Bol PM: Phytochemistry of the genus Piper. Phytochemistry 1997, 46(4):591-673. 
33. Omisore NOA, Adewunmi CO, Iwalewa EO, Ngadjui BT, Adenowo TK, Abegaz BM, Ojewole JA, Watchueng J: Antitrichomonal and antioxidant activities of Dorstenia barteri and Dorstenia convexa. Braz J Med Biol Res 2005, 38(7):1087-1094.

34. Ngameni B, Watchueng J, Fekam BF, Keumedjio F, Ngadjui TB, Gut J, Abegaz $B M$, Rosenthal PJ: Antimalarial prenylated chalcones from the twigs of Dorstenia barteri var. subtriangularis. ARKNOC 2007, 13:116-123.

35. Kuete V, Ngameni B, Tangmouo GJ, Bolla JM, Alibert-Franco S, Ngadjui TB, Pagès JM: Efflux Pumps Are Involved in the Defense of Gram-Negative Bacteria against the Natural Products Isobavachalcone and Diospyrone. Antimicrob Agents Chemother 2010, 54(5):1749-1752.

doi:10.1186/1756-0500-5-299

Cite this article as: Voukeng et al:: Antibacterial and antibioticpotentiation activities of the methanol extract of some cameroonian spices against Gram-negative multi-drug resistant phenotypes. BMC Research Notes 2012 5:299.

\section{Submit your next manuscript to BioMed Central and take full advantage of:}

- Convenient online submission

- Thorough peer review

- No space constraints or color figure charges

- Immediate publication on acceptance

- Inclusion in PubMed, CAS, Scopus and Google Scholar

- Research which is freely available for redistribution 